\title{
The Intermediate Band Solar Cell: Progress Toward the Realization of an Attractive Concept
}

\author{
By Antonio Luque* and Antonio Martí
}

The intermediate band (IB) solar cell has been proposed to increase the current of solar cells while at the same time preserving the output voltage in order to produce an efficiency that ideally is above the limit established by Shockley and Queisser in 1961. The concept is described and the present realizations and acquired understanding are explained. Quantum dots are used to make the cells but the efficiencies that have been achieved so far are not yet satisfactory. Possible ways to overcome the issues involved are depicted. Alternatively, and against early predictions, IB alloys have been prepared and cells that undoubtedly display the IB behavior have been fabricated, although their efficiency is still low. Full development of this concept is not trivial but it is expected that once the development of IB solar cells is fully mastered, IB solar cells should be able to operate in tandem in concentrators with very high efficiencies or as thin cells at low cost with efficiencies above the present ones.

\section{Introduction}

The photovoltaic (PV) conversion of the solar energy is a clean ${ }^{[1]}$ way of producing electricity with high land-occupation efficiency (e.g., as compared to biomass) that for sustainability should (and most probably will) become a major source of electricity. ${ }^{[2]}$

Unfortunately, PV conversion is expensive. Novel cell concepts, able to produce an important increase of the efficiency, are probably necessary and, among them, we describe the one to whose development we have contributed more effort: the intermediate band (IB) solar cell. We present the challenges and difficulties related to IB solar cells but also the progress achieved since we presented the concept ${ }^{[3]}$ in 1997.

To make solar cells, two elements are required (Fig. 1a). On one side, a material with two energy levels or sets of levels should be present; this can well be a semiconductor with its valence band (VB) forming the set of lower energy levels and its conduction band (CB) forming the set of higher energy levels. In this system, solar photons can pump electrons from the VB to the CB and as a result become absorbed in the process. Once the electrons are in

[*] Prof. A. Luque, Prof. A. Martí Instituto de Energía Solar Universidad Politécnica de Madrid Avenida Complutense 30, 28040 Madrid (Spain) E-mail: a.luque@upm.es the CB, a selective contact to this band is required through which the electrons pumped to the $\mathrm{CB}$ flow outside the semiconductor forming an electric current at high potential (actually the electrochemical potential of the electrons in the $\mathrm{CB}, E_{\mathrm{FC}}$ ). Outside the cell, these electrons lose their energy by providing a power source, for example by powering an electric motor. The electrons then, at a lower energy, are fed back to the semiconductor VB trough another selective contact (at the electrochemical potential of the electrons in the $\mathrm{VB}, E_{\mathrm{FV}}$ ). In conventional solar cells, doping the semiconductor with acceptor and donor impurities so forming a pn-junction makes the selective contacts. Silicon cells, as well as thin film cells of several types, follow this fundamental scheme.

The solar cells just described present a fundamental efficiency limitation that was determined by Shockley and Queisser ${ }^{[4]}$ on the basis of detailed balance arguments. This limit, corrected to take into account operation under full concentration ${ }^{[5]}$ (the one providing isotropic illumination on the cell with the radiance corresponding to the sun's photosphere), is $40.7 \%$. The main reason that underlies this value is that only photons with an energy level close to that of the semiconductor bandgap are effectively converted. Photons with lower energy are lost (the semiconductor is transparent to them) and of the photons with higher energy, only a part of this energy, i.e., that equals the bandgap energy (actually, this value multiplied by a Carnot factor) is at best converted.

But other concepts may lead to very high efficiency. Among them, the IB solar cell, ${ }^{[3]}$ which will be the main topic of this article, has raised considerable attention. IB solar cells can be used to increase the efficiency of low-cost solar cells but they also have the potential of operating with very high efficiency. High efficiency cannot easily be conciliated with low cost. In any case, it is desirable to liberate solar cell manufacturing from cost constraints. ${ }^{[6]}$ This can possibly be achieved by the use of concentrators because these optical systems increase the energy cast on each expensive solar cell, making its use cost-effective. Although there are challenges to overcome, very low electricity cost is expected if the efficiency and concentration factor are high enough. ${ }^{[7]}$ Operation at 1000 suns (i.e., at $100 \mathrm{~W} \cdot \mathrm{cm}^{-1}$ ) is today being considered for multijunction (MJ) solar cells and could also be used for the novel concepts developed here. 
a)

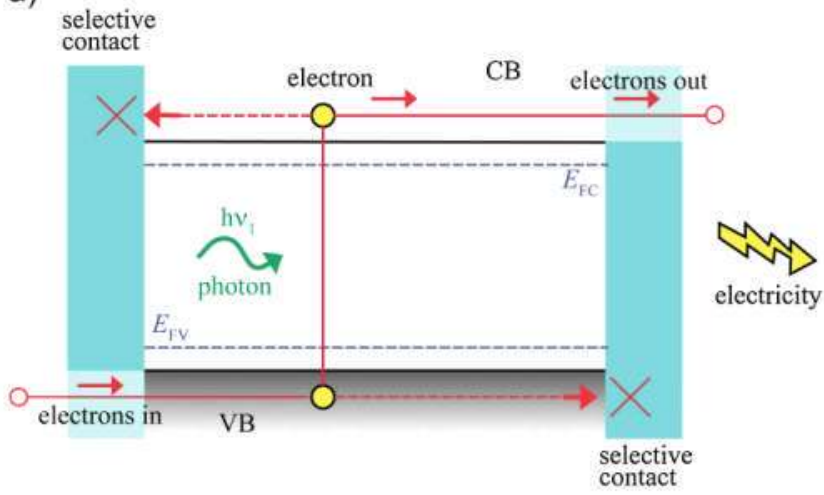

b)

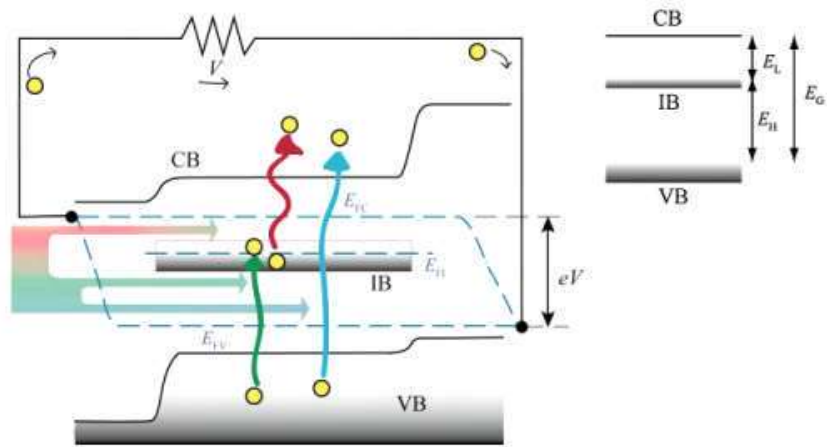

Figure 1. a) A solar cell consists of a material with two energy levels ( $C B$ and $V B$ ) and selective contacts to these levels. Photons pump electrons from the $V B$ to the $C B$ that are supplied at high energy to the external circuit. Then, with their energy lost by producing the required output, the electrons return to the VB. b) Photon absorption process and quasi-Fermi level split in an IB solar cell. The basic structure of an IB solar cell consists of an IB material sandwiched between conventional semiconductors of the $\mathrm{p}$ - and $\mathrm{n}$-type. These conventional semiconductors act as selective contacts to electrons and holes, and prevent the IB from contacting the external electrodes. The IB divides the total bandgap $E_{\mathrm{C}}$ in two sub-bandgaps, $E_{\mathrm{L}}$ and $E_{\mathrm{H}}$, where $E_{\mathrm{L}}$ is the energy level with the lowest value, usually the one that separates the IB from the CB (inset). Photons from the sun, when absorbed, pump electrons from the IB to the $C B$ (red vertical arrow), from the VB to the IB (green arrow), and from the VB to the CB (blue arrow). Since carrier relaxation between bands is a much slower process than carrier relaxation within bands, a distinct quasi-Fermi level is associated with each of the bands: $E_{\mathrm{FC}}, E_{\mathrm{FV}}$, and $E_{\mathrm{Fl}}$ (dashed blue lines). Since the output voltage $\mathrm{eV}$ is given by the split between electrons and hole quasi-Fermi levels, this voltage is still limited by the total bandgap $E_{\mathrm{G}}$.

\section{The Intermediate Band Solar Cell Concept}

\subsection{Increasing the Current While Preserving the Voltage}

\subsubsection{Double Band Absorption IB Solar Cells}

An IB solar cell ${ }^{[3,8]}$ consists of an IB material situated between two conventional semiconductors, $\mathrm{n}$ - and $\mathrm{p}$-type, that play the role of selective contacts to $\mathrm{CB}$ and $\mathrm{VB}$ electrons, respectively. As shown in Figure 1b, the IB material has a band of states inside the bandgap between the CB and the VB. In this way, photons with

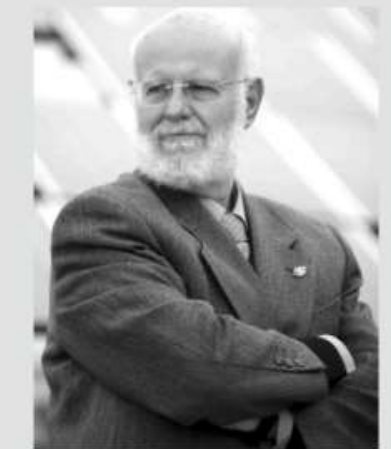

Antonio Luque (b. 1941) obtained his Doctor Ingeniero de Telecomunicación degree at the Universidad Politécnica de Madrid. He is Full Professor of Electronic Technology at the same university since 1970 and founder of the Institute of Solar Energy in 1979. His research activity is devoted to photovoltaics. In 1981, he created the solar cells company Isofotón with a turnover of 297 million Euro in 2007 . He has been the recipient of several important awards for photovoltaic research.

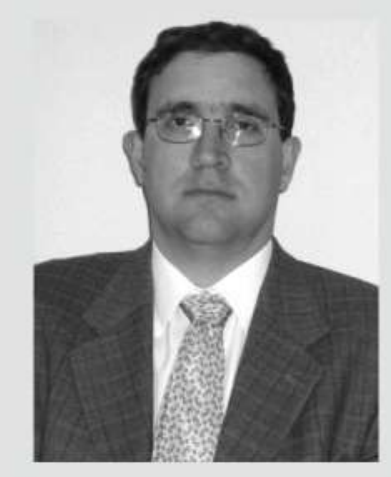

Antonio Martí got his Ph.D. by the Universidad Politécnica de Madrid in 1992. Full Professor of Electronic Technology at the same university since 2007. Since 1997 he is collaborating with Prof. Luque on the development of intermediate band solar cells.

less energy than the one necessary to pump an electron from the $V B$ to the $C B$ can be absorbed by transitions that pump an electron from the VB to the IB (green arrow) and from the IB to the $\mathrm{CB}$ (red arrow). Thus a full $\mathrm{VB} \rightarrow \mathrm{CB}$ electron transition (also called electron-hole pair generation) can be completed by means of two photons of energy below the bandgap. This mechanism adds to the conventional one, pumping electrons from the VB to the CB by means of a single photon (blue arrow).

Obviously, the mechanism just described increases the cell photocurrent because photons of lesser energy are collected. Nevertheless, increasing the current of a solar cell is conceptually easy. It suffices to choose a lower bandgap semiconductor for producing the cell. However, this choice would also reduce the voltage that, except when lasing, cannot exceed the semiconductor bandgap. Since the electric power is the product of the current times the voltage, there is an optimum bandgap that is located around that of the silicon bandgap $(1.12 \mathrm{eV})$.

The key point is then to increase the photocurrent without reducing the voltage (too much). To this end, three electrochemical potentials, called quasi-Fermi levels by device physicists, should appear in the IB material, two of them associated with the VB and CB same as in conventional solar cells, and the third one associated with the IB (Fig. 1b). The voltage extracted from the cell is the difference between the $C B$ and VB quasi-Fermi levels at the $\mathrm{n}$ - and p-contacts, respectively (divided by the charge of the electron). 
a)

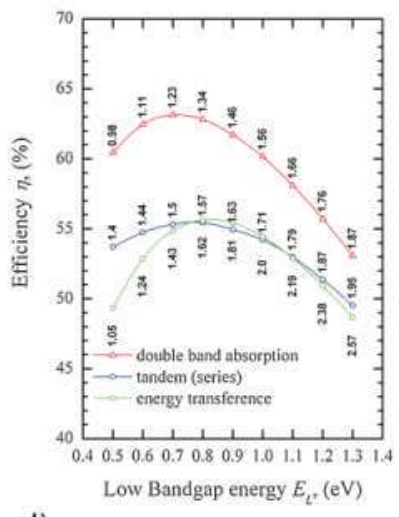

d)

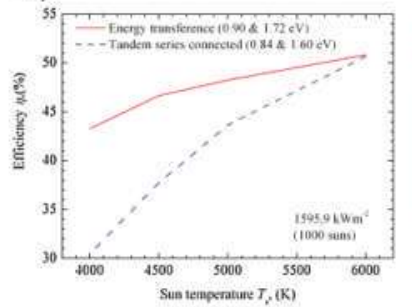

b)

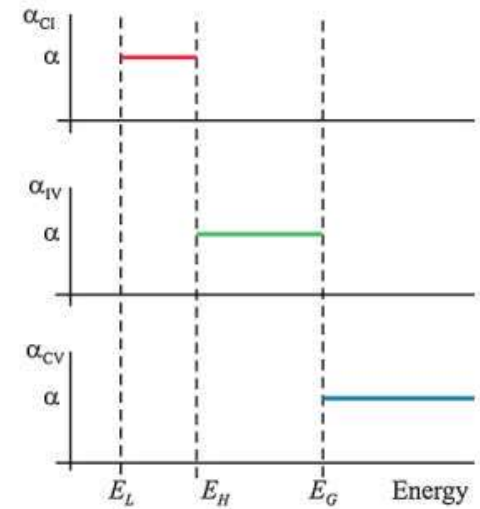

e)

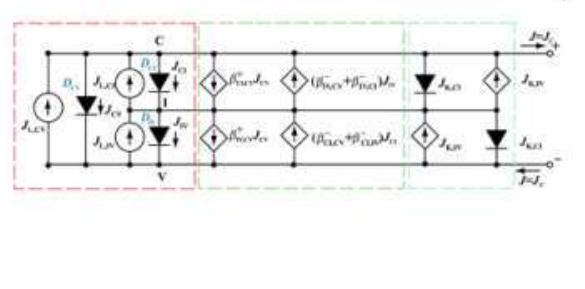

c)

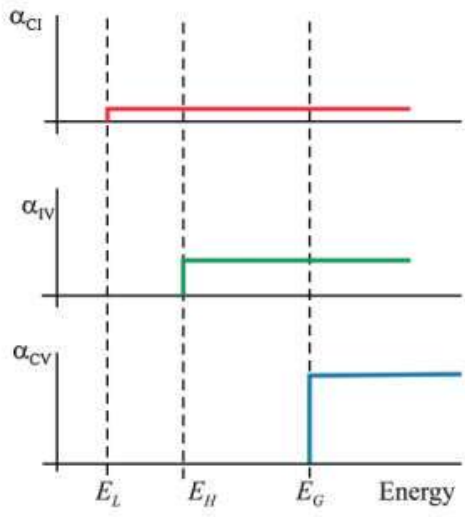

f)

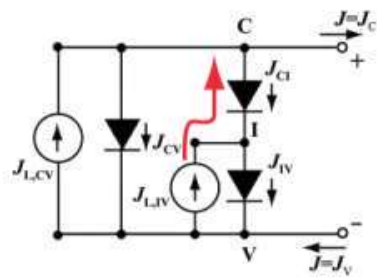

Figure 2. a) Limiting efficiency of the IB solar cell as a function of the lower bandgap energy, $E_{L}$ in the case of optimal VB $\rightarrow I B$ and $I B \rightarrow C B$ absorptions (red line or "double band absorption"). The numbers in the plot indicate the optimum value for the second bandgap, $E_{H}$ (adapted with permission from [3], copyright 2003, IEEE). In (b) and (c), the absorption coefficient related to the IB $\rightarrow$ CB transition is shown in red, the one associated with the VB $\rightarrow$ IB transition in green, and the one associated with the VB $\rightarrow$ CB transition in blue (adapted with permission from [10], copyright 2008, Elsevier). The blue line in the (a) plots, for reference purposes, corresponds to the limiting efficiency of a tandem configuration of two single-gap solar cells connected in series. In this case, the numbers on the $x$-axis corresponds again to the value of the low bandgap cell and the data in the curves correspond to the value of the high-bandgap cell. The green line in (a) corresponds to the limiting efficiency of an IB solar cell operating under the "energy transfer" concept. For all cases plotted in (a), the values of the bandgap appearing next to the plotted curves have been optimized to achieve maximum efficiency. d) The efficiency for the "energy transference" case as a function of the sun temperature for the cell with optimum bandgaps (red line) (adapted with permission from [11], copyright 2008, Elsevier). The results are also compared with the optimum cell corresponding to a tandem of two solar cells connected in series (blue line). In all cases, although the temperature of the sun changes, the input power is kept constant at $1595.9 \mathrm{~kW} \mathrm{~m}^{2}$, equivalent to an irradiance of 1000 suns of a black body sun at $6000 \mathrm{~K}$. e) A circuit-like model for the IBSC (note that current arrows actually represent electron flows, opposite to actual currents, and voltages represent electron quasi-Fermi levels in eV, opposite to actual voltages). The elements in the red box model generation (current generators) and recombination (diodes) processes between bands; the elements in the green box model phenomena related to the re-absorption of the photons created in recombination mechanism; the elements in the blue box model energy transfer phenomena (adapted with permission from [12], copyright 2004, AIP). The voltage at the points C, I, and V show the quasi-Fermi level positions (in eV). f) A simplified version of this circuit-like model when there is no energy transfer, the absorption coefficients do not overlap and there is no absorption of photons related to transitions from the IB to the $C B\left(U_{L, C l}=0\right)$. In this case (red arrow), the extraction of current due to absorption of photons from the VB to the IB implies that the diode $\mathrm{Cl}$ is reverse-biased.

The limiting efficiency of the IBSC concept for full concentration is $63.2 \%{ }^{[3]}$ (see Fig. 2a) in comparison to the Shockley-Queisser limit of $40.7 \%^{[4,5]}$ for a conventional single-gap solar cell under the same operating conditions. Hence, in both cases, the efficiency refers to the case in which any non-radiative recombination is suppressed, carrier mobilities are infinite (no ohmic losses), illumination comes from an isotropic gas of photons at $6000 \mathrm{~K}$ (taken, not accurately, as the sun's photosphere temperature) and the cell lattice is at $300 \mathrm{~K}$. To achieve this illumination, an ideal concentrator producing a concentration of about $40050 \mathrm{X}$ is assumed. ${ }^{[9]}$

\subsubsection{Photon Selectivity}

The absorption of the photons emitted as a consequence of a carrier recombination process between a given couple of bands by a different couple is detrimental to the performance of the IB solar cell. This is avoided if the absorption spectra between two bands $(\mathrm{IB} \rightarrow \mathrm{CB}, \mathrm{VB} \rightarrow \mathrm{IB}, \mathrm{VB} \rightarrow \mathrm{CB}$ ) does not overlap with that of another pair ${ }^{[3]}$ as represented in Figure $2 \mathrm{~b}$. An equivalent condition (Fig. 2c) can be obtained in practice if the three spectrums overlap but there is a large difference in the absorption coefficients in the range of wavelengths where they overlap. ${ }^{[13]}$ If no light confinement is used, this last case may require a very thick IB material to allow the absorption of the photons with the weakest absorption coefficient.

\subsubsection{Light Confinement}

Light confinement consists of increasing the ray path of the photons. ${ }^{[14]}$ Light confinement can be achieved by texturizing the cell surface. Photons cannot escape easily because they are reflected back by total internal reflection. The enlargement of the light rays can reach up to $4 n_{\mathrm{r}}^{2}\left(n_{\mathrm{r}}\right.$ : semiconductor index of 
refraction), that is, about 50 in many semiconductors, although $1 / 3$ of this value is a good practical figure.

It might also be produced by diffraction structures that deflect the light almost parallel to the surface. This concept has been studied for solar cells ${ }^{[15,16]}$ and its performance limit has been published ${ }^{[17]}$ For the calculation of this limit, the angular dispersion caused by the sun angular aperture, usually increased if the cell is to be used under concentrated sunlight, has been taken into account. This sets a limit to the length of the path that can be achieved by diffraction and this limit is found to be 320 times the IB material thickness when operating without concentration or 40 times when a reasonable concentrator is used. This approach is wavelength-dependant: the path length decreases to less than $90 \%$ when the wavelength is within $10 \%$ of the designed one. This is probably enough if we take into account that the path lengths have to be increased only for the less absorbed photons and not for all the wavelengths.

\subsubsection{Energy Transference in IB Solar Cells}

Pure energy transference between electrons in the IB, by impact ionization (whose recombination counterpart is an Auger mechanism) or any other mechanism, can also lead to satisfactory IB solar cells. ${ }^{[11,18]}$ In this mode, two sub-bandgap photons pump two electrons from the VB to the IB. Then, one of the electrons returns to the VB and transfers its energy to the other electron in the IB that is so pumped to the CV. The limit efficiency under this mode of operation is somewhat smaller (Fig. 2a).

One interesting feature of this mode of operation is its smaller sensitivity to the solar spectrum (Fig. 2d). In addition, the conditions sets for the absorption coefficients in order to achieve the photon selectivity described above become relaxed.

\subsubsection{General Equivalent Circuit}

Figure 2e shows the equivalent circuit for an IB solar cell. ${ }^{[12]}$ This equivalent circuit includes (inside the red box) three current generators and three diodes. The diodes represent the recombination associated with radiative transitions. Their reverse saturation current, as well as their ideality factor, can be increased to count non-radiative recombination processes. The current generators inside the red box represent the photocurrent generated by the absorption of photons from the sun. The current generators inside the blue box represent the photocurrent generated as a consequence of the re-absorption of photons emitted as a consequence of radiative recombination processes. For instance, one photon emitted by an IB $\rightarrow V B$ transition can be re-absorbed by an IB $\rightarrow \mathrm{CB}$ transition. The existence of this process, for example, is taken into account by the $\left(\beta_{\mathrm{CI} . \mathrm{IV}}^{-}+\beta_{\mathrm{CV} . \mathrm{IV}}^{-}\right) J_{\mathrm{CV}}$ current generator. The elements in the green box correspond to the circuital representation of the energy transference mechanism described above.

This equivalent circuit assumes constant quasi-Fermi levels. If this is not the case, in the whole IB material the circuit can be applied to narrow IB material layers where the quasi-Fermi levels can be considered constant.

\subsubsection{A Thermodynamic Requirement}

It is important to understand that a fundamental condition to keep the voltage of a solar cell that uses sub-bandgap photon absorption (as it is the IB solar cell) above the energy of the lowest photon absorbed is the need for the absorption of at least a second photon that provides the necessary free energy. This is a thermodynamic requirement. ${ }^{[19-21]}$ Sometimes, thermal escape has been claimed as the sufficient mechanism to pump electrons from the IB (or the alternative name given to this level by other authors) to the CB. This is indeed possible, but if this is the case (see Fig. 2f), in the absence of $\mathrm{IB} \rightarrow \mathrm{CB} \quad \mathrm{U}_{\mathrm{L}, \mathrm{IC}}$ ) photo-generation and in the absence of impact-Auger mechanisms (DK) the thermal escape (the reverse current of the DCI diode) will not happen unless the quasi-Fermi level in point $I$ is above the quasi-Fermi level in point $C$. This is the same as saying that the IB quasi-Fermi level is above the CB quasi-Fermi level and implies that the introduction of an IB does not increase but reduces the cell voltage that now becomes limited by the $E_{\mathrm{H}}$ sub-bandgap (Fig. 1b).

\subsection{Impurity Levels Versus Bands}

\subsection{Multiple Phonon Emission}

A question arising when an IB solar cells is proposed is the following: impurity deep levels are known to introduce a strong Shockley-Read-Hall ( $\mathrm{SRH}$ ) recombination, that is, a non-radiative downward transition that is detrimental to solar cell performance; why should it be different in the IB solar cell?

The relatively long lifetimes of the excited electrons in semiconductors are based on the scarcity of particles that are of high enough energy to collect the energy delivered in the recombination. Radiative recombination-where these particles are photons - is just a detailed balance counterpart of the light absorption process, which in solar cells is fundamental. In this sense it is unavoidable and a solar cell only limited by such recombination is ideal. On the contrary, non-radiative recombination is, in principle, avoidable and for ideal performance it must be suppressed.

The research on the physics of non-radiative recombination has been a long-standing question in semiconductor physics. Non-radiative recombination can occur by transferring energy to other electrons-Auger recombination-or to phonons. Auger recombination occurs mainly in heavily doped regions such as the emitters. For less doped materials, recombination assisted by phonons (quanta of lattice oscillations) is dominant but, still, the low energy of the phonon (tens of meV) as compared to the bandgap requires, at a first glance, that some kind of improbable multiphonon mechanism for a recombination to occur. It is, however, well established that impurities leading to deep levels are the cause of the $\mathrm{SRH}$ recombination.

We believe ${ }^{[22]}$ that the most frequent cause of non-radiative $\mathrm{SRH}$ recombination in the bulk of a semiconductor (in the surface, cascaded mechanisms might be dominant) is the so-called lattice relaxation multiple-phonon emission mechanism (MPE). ${ }^{[23]}$

In a semiconductor, electrons in the CBs and VBs are characterized by Bloch wavefunctions extending across the whole crystal. In contrast, the electrons in the deep traps are characterized, when the traps are enough diluted, by localized 
wavefunctions. In this situation, if a transition is produced from an electron in, for example, the CB to the localized state at the impurity, then there is a large, swift charge movement and the charge of an electron formerly distributed across the whole crystal becomes suddenly closely packed around the impurity. This causes the impurity to be strongly out of equilibrium in the crystal lattice and consequently the impurity and the neighboring atoms start vibrating heavily in search of a new equilibrium. A "breathing mode" has been produced.

The transition is not improbable because, in such a non-equilibrium position, the energy level of the filled trap is situated at an energy similar or very close to that of the electrons in the CB, as can be seen in the configuration diagram of Figure $3 a$ (point A). Therefore, the transition conserves the energy either strictly speaking, or, at worst, with the emission or absorption of a single phonon.

The violent vibration induced in the impurity by the transition is dampened to its thermal value by the successive delivery of phonons through ordinary phonon-phonon and phononelectron interactions. The number of phonons delivered to complete the transition is the so-called Huang-Rhys factor ${ }^{[25]}$ that can be in the range of 10 or 12 on many SRH capture processes.

The transition (point B in Fig. 3a) of the localized electron wavefunction in the impurity's deep level to the extended state in the VB (hole capture) involves again a strong charge movement and the impurity becomes again out of equilibrium. A new breathing mode is produced that is dampened as before, involving a second Huang-Rhys factor.

\subsubsection{Suppressing Non-radiative Recombination}

Increasing the density of traps, so that the Mott transition ${ }^{[26]}$ (or any other delocalization mechanism) is produced, will cause the impurity wavefunctions not to be localized anymore and become extended. Mott justifies this fact as follows: Removing an electron from the impurity involves leaving the impurity positively charged (at least with respect to the former state) and this implies the appearance of a Coulomb attraction. But this attraction becomes weakened by the presence of other impurity-electron wavefunctions that produce screening. This screening leads to a potential energy $-e^{2} \exp (-\lambda r) / 4 \pi \varepsilon_{0} r$ (units in SI) where the screening factor $\lambda$ is given by $\lambda^{2} \cong e^{2} N_{\mathrm{T}} / 2 \varepsilon \varepsilon_{0} k T$. The damping factor increases with the density of impurities $N_{\mathrm{T}}$.

When the density of impurities is such that $a_{\mathrm{H}} \lambda>0.888$, $a_{\mathrm{H}}=4 \pi \varepsilon \varepsilon_{0} \hbar^{2} / m_{0} e$ being the Bohr radius (no effective mass is used here because the electrons concerned do not move in a band), the Coulomb potential ceases to have a localized state and the electron is free to escape from the impurity. In this case, for all the impurities, an extended state is produced with an eigenfunction that is shared among all the impurities. In the case of periodic location of the impurities, this eigenfunction is a Bloch function but this is not relevant in our argumentation. According to the preceding model, the value for this delocalization to occur is $N_{\text {Tcrit }}=5.9 \times 10^{19} \mathrm{~cm}^{-3}$ at $300 \mathrm{~K}$ when the IB quasi-Fermi level crosses the IB.

When the delocalization has occurred, any transition from the $\mathrm{CB}$ to the impurity band will not involve a large charge movement in the space and therefore would not imply a strong
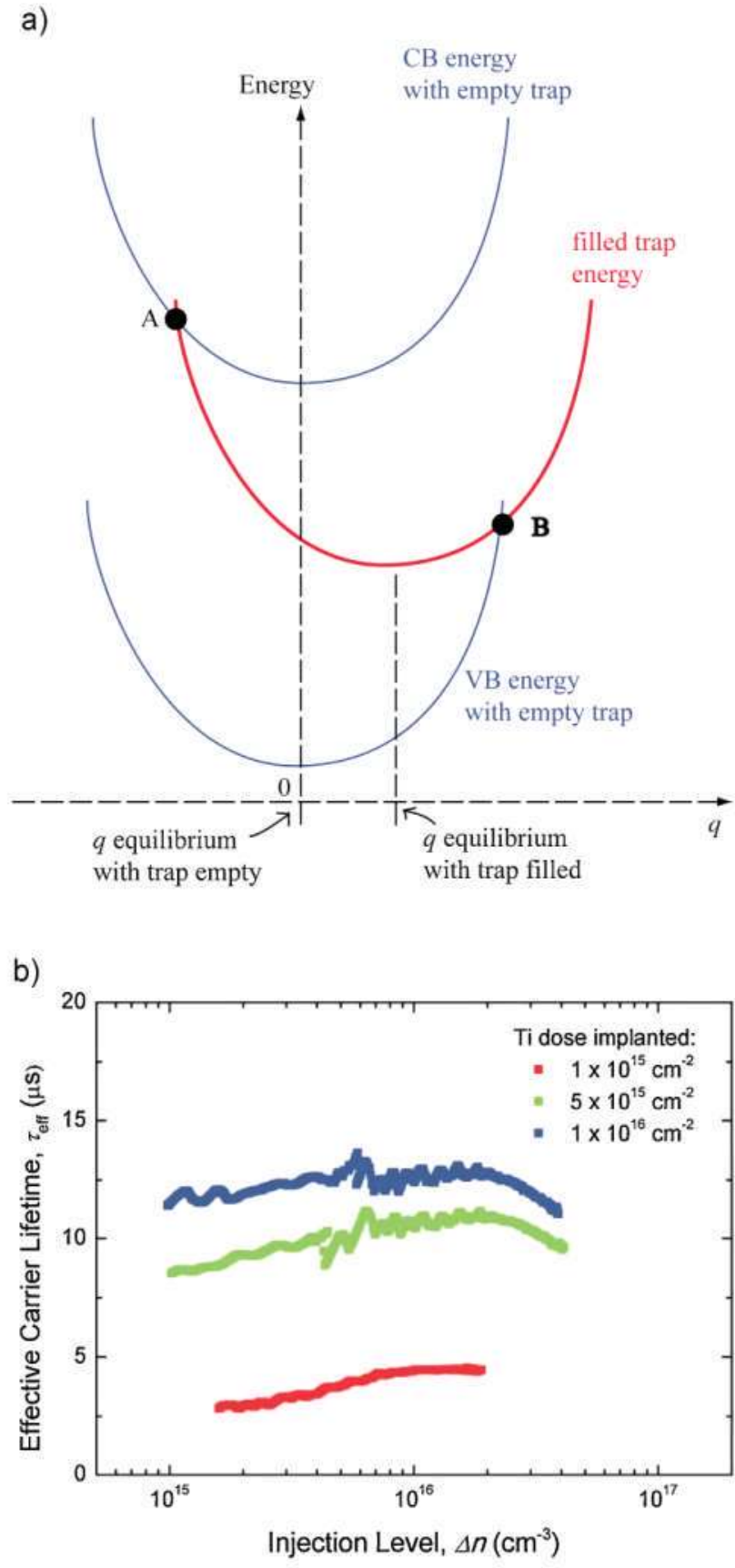

Figure 3. a) Configuration diagram illustrating the potential energy of the semiconductor nuclear equation (including both electrons and nuclei) per electron along the line of maximum potential slope parameterized by $q$ (adapted with permission from [22], copyright 2006, Elsevier). b) Effective lifetime measured by the stationary photo-conductance technique on a silicon wafer where high titanium doses have been implanted. Higher doses lead to longer lifetimes, against the normally expected behavior and accordingly to the model of non-radiative recombination suppression (reproduced with permission from [24], copyright 2009, AIP).

disequilibrium. As a consequence, the impurity's potential energy is not shifted laterally (transition point A in Figure 3a, if it exists, appears at very high energies) and the transition between the CB and the deep level cannot occur. In other words, the SRH recombination has been prevented. 
This actually happens when the impurities are close enough to start interaction-forming bands and it ultimately explains why no recombination is to be expected from IBs as those described in the preceding section. It sets a conceptual difference between what has been called "Impurity Photovoltaics"[27] and "Intermediate Band Solar Cells".

\subsubsection{Experimental Evidence}

An experimental confirmation of this fact has recently been obtained in Si samples that were heavily doped with Ti. The samples were prepared ${ }^{[28]}$ by heavy ion implantation (with densities above $10^{21} \mathrm{~cm}^{-3}$ ) followed by a pulsed laser melting process that restores crystal quality. Ti produces deep levels in silicon and leads to strong recombination, ${ }^{[29-31]}$ and is thus known to be a minority carrier lifetime killer. The effective lifetime has been measured by the stationary photo-conductance technique $^{[32]}$ (Fig. 3b) and longer lifetimes have been found for the samples with the higher $\mathrm{Ti}$ doses ${ }^{[24]}$ implanted in agreement with our predictions. ${ }^{[22]}$

\section{Quantum Dot Intermediate Band Solar Cells}

\subsection{The Concept}

Quantum dots (QDs) are droplets or nanocrystals of a material immersed in a matrix material usually with a higher bandgap. Such materials are often called dot and barrier materials, respectively. QDs have been proposed $^{[33,34]}$ as one of the means for taking to practice the IB solar cell. The IB would in this case typically arise from the confined states of the electrons in the CB tridimensional potential wells originated by the CB offset between the dot and barrier material (Fig. 4a).

For IB solar cells, QDs and not other low-dimensional structures such as quantum wells or wires are necessary (or at least preferable) because QDs form tridimensional potential wells that ideally isolate the IB from the CB through a true zero density of states. However, the practical achievement of this condition needs to be reconsidered later.

In order to facilitate the absorption of photons that cause transitions from the IB to the $\mathrm{CB}$, the IB has to be partially filled with electrons so that there are enough electrons to be promoted to the CB. This can be achieved by introducing some $n$-type doping in the barrier $^{[34]}$ region at a concentration that approximately equals the dot concentration ( $\delta$-doping layers in Fig. 4b).

In our early studies, ${ }^{[37]}$ we tried to identify proper dot and barrier materials that are able to produce the optimal bandgaps of $0.71,1.24$, and $1.95 \mathrm{eV}$. We assumed QDs of spherical symmetry and used the effective mass approximation to calculate the materials and dot size. We proposed $\mathrm{In}_{0.58} \mathrm{Ga}_{0.42} \mathrm{As}$ as dot material with a dot diameter of $5.8 \mathrm{~nm}$ and $\mathrm{Al}_{0.4} \mathrm{Ga}{ }_{0.6} \mathrm{As}$ as barrier material although these values were modified to $\operatorname{In}_{0.55} \mathrm{Ga}_{0.45} \mathrm{As}$ as dot material with a dot diameter of $9.4 \mathrm{~nm}$ and $\mathrm{Al}_{0.44} \mathrm{Ga}{ }_{0.56} \mathrm{As}$ as barrier material when strain considerations were taken into account.

Quantum wells, mentioned above, are nanolayers of a lower bandgap semiconductor inside the matrix material and electrons result confined in one direction only; therefore, there is a continuous of states joining the confined level and the semiconductor band associated with the transversal velocity of the electrons that is not quantified. In consequence what the quantum well essentially does is to reduce the semiconductor bandgap (with respect to that of the barrier material) and therefore produce a new semiconductor with a modified
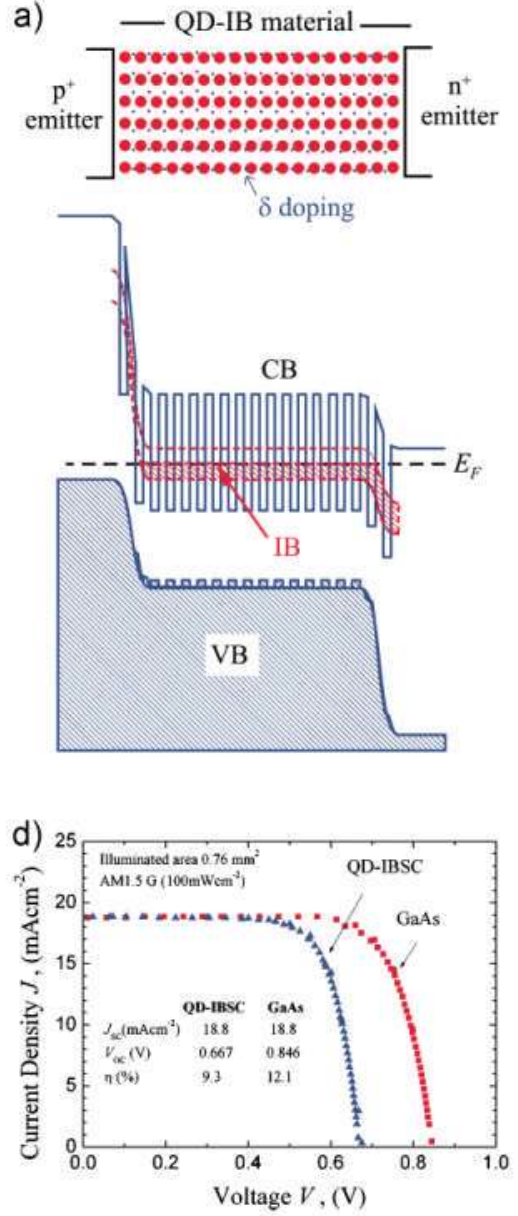

Figure 4. a) Simplified bandgap diagram of an IB solar cell manufactured using QDs (adapted with permission from [10], copyright 2008, Elsevier). b) Details of the layer structure of a complete QD IB solar cell (adapted with permission from [12], copyright 2004, AIP). c) TEM image of InAs QDs on a GaAs barrier (reproduced with permission from [35], copyright 2007, AIP). d) Typical current-voltage characteristic (red) under illumination compared to a GaAs reference (blue) (adapted with permission from [36], copyright 2006, AIP). e) Quantum efficiency of a QD-IBSC compared to a GaAs reference cell (adapted with permission from [10], copyright 2008, Elsevier). 
bandgap. They can be used to tune the bandgap of the cells in M) approaches and can lead to produce very good solar cells. ${ }^{[38,39]}$ However, it has to be stressed that in the QW cells a separation of the QW and CB quasi-Fermi levels is extremely difficult and therefore a voltage above the absorption threshold, that is, the main purpose of the IB solar cell, cannot appear (Section 2.1.6).

\subsection{Realization}

The dot/barrier combination calculated above for the IB solar cell might be closer to the optimum but their technology, if possible, is not so well mastered. Much more experience is available for InAs dots in a GaAs matrix. First prototypes were manufactured based on this combination ${ }^{[12]}$ by the group of Colin Stanley at the University of Glasgow in cooperation with us (see Fig. 4b) within the framework of the FULLSPECTRUM project. The basic structure of these cells consisted of 10 layers of InAs/GaAs QDs sandwiched by $p$ and $n$ GaAs emitters and is grown by molecular beam epitaxy MBE under the Stranski-Krastanov growth mode. Under this growth mode, a layer of dot material latticemismatched to the barrier - grows first. When 2-3 monolayers are grown, this layer, forced by the accumulated stress, breaks and shrinks into droplets (the QDs). Nevertheless, a very narrow dot-material layer, called the wetting layer, remains unbroken forming a quantum well. This technique is relatively simple and cheap.

As visible in Figure 4c, these QDs do not have spherical symmetry. They look more like a lens or a truncated pyramid with a base length of about $10 \mathrm{~nm}$ and a height of about $3 \mathrm{~nm}$, dimensions that may be very dependant on the growth conditions. In this case, dots are rather close in the vertical direction although they are more spaced and irregularly located in the horizontal plane. Quantum calculations seem to show that the wavefunctions overlap ${ }^{[40]}$ in the vertical direction, which also leads to a delocalized behavior as suggested by the third-derivative shape that has been observed in the QD photo-reflectance spectrum. ${ }^{[1]}$ This delocalized behavior is probably limited to filaments along the QD columns observed in the figure and may lead to a (weak) vertical conduction, which is considered helpful. ${ }^{[42]}$

Figure $4 \mathrm{~d}$ shows the typical current-voltage characteristic of QD-IBSCs compared with that of a test GaAs sample possessing the same internal structure but lacking the QDs. As can be seen, the photogenerated current appears to be approximately the same in both cases. However, an examination of the quantum efficiency of the cells (Fig. 4e) reveals that this is not the case. The QD IB solar cells exhibit an extended response for photon energies lower than the bandgap. The contribution to the total current of the cell resulting from below bandgap energy photons is small (1\%) revealing a poor absorption provided by the QDs that is unable to overcome an open-circuit voltage loss.

The efficiency of this cell is not better than the GaAs cell to which it has been compared. The question of whether practical QD IB solar cells will be possible, will be discussed below. Anyway, the InAs/GaAs cell has been very practical to experimentally check the basic principles that induced the research on IB solar cells.

\subsection{Experimental Proof of the Intermediate Band Solar Cell Concept}

\subsubsection{Proof of a Two-Photon Absorption Mechanism for $V B-C B$ Pumping}

The appearance of a sub-bandgap photocurrent was clearly proven by quantum efficiency measurements since the fabrication of the first IB solar cells ${ }^{[12]}$ (see Fig. 4e). However, doubts existed about whether the $\mathrm{IB} \rightarrow \mathrm{CB}$ transition could be explained by a second photon being absorbed at this absorption band or by some other mechanism such as for example energy transference, ${ }^{[1]}$ thermal escape, field-assisted tunnel from IB to $C B$ in space charge regions or even a mixture of several of these mechanisms. The involvement of a second photon is important since, as it has been discussed, thermal escape and also tunneling allows the extraction of below bandgap photocurrent but does not allow voltage preservation because the free-energy provided by the second photon is lacking. An experiment was designed and performed, ${ }^{[43]}$ which demonstrated that twophoton $\mathrm{VB} \rightarrow \mathrm{CB}$ pumping is actually taking place. In this experiment, the cell is set at a very low temperature $(4-80 \mathrm{~K})$ to minimize thermal escape while the photocurrent produced by a monochromator is recorded as it scans different wavelengths. Then, an IR broadband light source is filtered by a low-energy band-pass filter insuring that only IB $\rightarrow$ CB transitions can be produced. This IR source is chopped (see Fig. 5a) and a lock-in amplifier collects the photocurrent produced at the chopper frequency only (Fig. 5b). This current is absent if the IR light is switched off and also when the continuous light from the monochromator does not have sufficient energy to pump an electron from the VB to the IB. This happens for energies below $1 \mathrm{eV}$. For energies above $1.9 \mathrm{eV}$, no synchronous photocurrent is extracted either, the reason being that photons from the monochromator are absorbed at the cell emitter and do not reach the QD region.

Although the current measured in the lock-in is rather small, the actual photocurrent associated with transitions from the IB to the CB might be larger. In reality, the chopped current (neglecting second-order effects) runs partly via the $D_{\mathrm{CI}}$ diode (Fig. 2), i.e., reverse-biased but with high saturation current (because the $E_{\mathrm{L}}$ bandgap is small) and this current is not measured as an external current. $^{[45]}$

\subsubsection{Proof of Three Quasi-Fermi Levels Splitting}

The highest electroluminescence peak of the cells $\left(\varepsilon_{2}\right.$ peak in Fig. $5 c)$ corresponds to photon emission from the IB to the VB. The smallest peak $\left(\varepsilon_{1}\right)$ corresponds to transitions from the $C B$ to the VB (the other peaks in the plot are associated with the existence of additional energy levels in the IB). The relative intensity between both peaks, combined with data gathered from quantum efficiency measurements, allows the determination of the quasi-Fermi level split $\left(\mu_{\mathrm{CI}}=E_{\mathrm{FC}}-E_{\mathrm{FI}}\right)$ that exists between the IB and the CB. This splitting has been measured ${ }^{[44]}$ (in the dark, in a direct-biased cell) and is of the order of the one shown in Figure $5 \mathrm{~d}$. This proves the possibility of the separation between quasi-Fermi levels that is a necessary condition for preserving the output voltage of the cell. 
a)

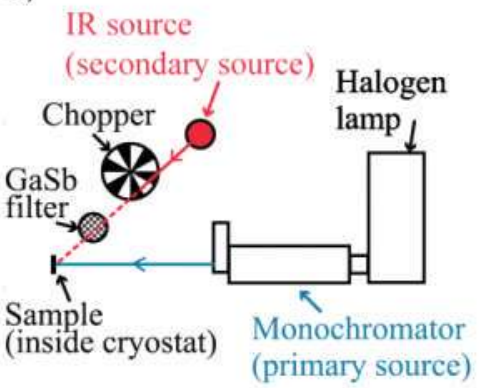

c)

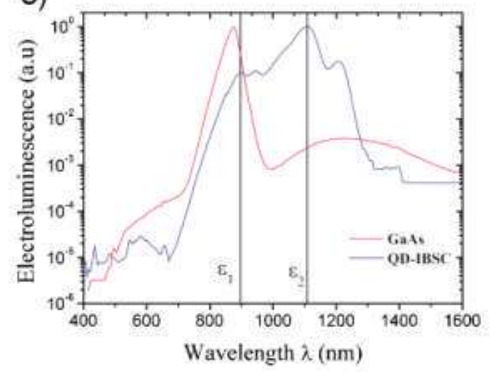

b)

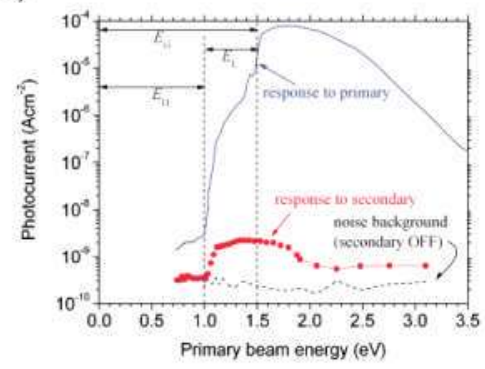

d)

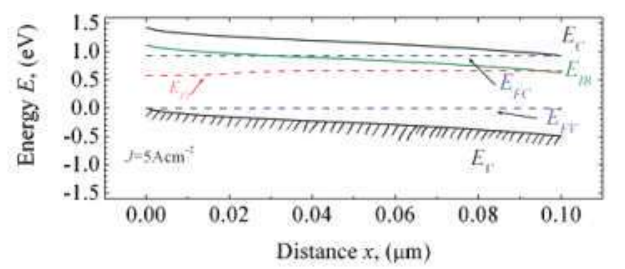

Figure 5. a) Setup of the experiment for demonstrating two-photon generation phenomena in an IB solar cell (adapted with permission from [43], copyright 2006, APS). b) Results of the experiment (adapted with permission from [43], copyright 2006, APS). The blue curve is the photocurrent response of the cell to the light from the monochromator (primary source) when only this source is ON. The red curve is the response to the chopped light (secondary source), that is, to the light that is only able to pump electrons from the IB to the $C B$, as a function of the energy of the photons of the primary beam. The noise background (black curve), that is, the signal existing in the curve devoted to measure the response to the IR (secondary) source when this source is OFF, is also plotted for reference. c) Electroluminescence spectrum of a QD IB solar cell compared to that of the GaAs control cell when both cells are biased at $5 \mathrm{~A} \cdot \mathrm{cm}^{-2}$. The plot shows the photon emission associated with transitions from the IB to the VB $\left(\varepsilon_{2}\right)$ and from the CB to the VB $\left(\varepsilon_{1}\right)$ (adapted with permission from [44], copyright 2006, AIP). d) Results of the modeling and the fit to the experimental results (adapted with permission from [36], copyright 2006, AIP).

\subsection{Toward a Practical Quantum Dot Intermediate Band Solar Cell}

\subsection{The Potential of Quantum Dot InAs/GaAs IB Solar Cells}

As mentioned before, the IB solar cell made of InAs QDs in a $\mathrm{GaAs}$ barrier material has been selected as the workhorse to test the principles of operation of the IBSC due to the fact that it is a rather well-known system. However, the bandgaps in this system (around $0.30,1.02$, and $1.30 \mathrm{eV}$ ), whose main bandgap differs from the one of the GaAs (see Section 3.4.3), are far from the ideal ones $(0.71,1.24$, and $1.95 \mathrm{eV}$, see Fig. 2a).

A consequence is that the detailed balance efficiency limit at 1 sun of the QD IB cell made of these materials is lower ${ }^{[10]}(30.2 \%)$ than the one $(31.0 \%)$ of a single gap solar cell of $1.3 \mathrm{eV}$. However, it is interesting to note the fact that the detailed balance efficiency limit for the QD IB cells increases up to $51.6 \%$ in comparison to $36.7 \%$ for the $1.3 \mathrm{eV}$ single gap cell if we increase the concentration from 1 sun to 1000 suns for the same choice of bandgaps. The reason for this improvement is that, at high voltage (which occurs when cells are operated under concentrated light), the photon flux is increased by the concentration factor while the recombination path through the IB increases more slowly than the one associated with the $\mathrm{CB} \rightarrow \mathrm{VB}$ transitions (Fig. 6a). Therefore, at high concentrations, recombination is dominated by transitions from the CB to the VB (as in single gap solar cells) while the IB still provides the benefits of increased sub-bandgap photo-generation.

\subsubsection{The Issue of the Current}

Practical QD IB solar cells are far from the ideal characteristics derived from detailed balance analyses. First, the increase of current due to sub-bandgap photo-generation observed in the current-voltage characteristic is almost negligible (Fig. 4d). Since in the cell of the example only 10 layers of QDs were grown, the obvious reaction is to increase the number of layers. In collaboration with our partners at the University of Glasgow, cells with 50 layers were grown but the photocurrent of the cells was actually worse, ${ }^{[46]}$ although the sub-bandgap current was greatly increased, as expected. The reason was that the upper emitter was totally degraded by dislocations created by the stress produced when the QDs were formed. Although this stress is partially released when the QDs are formed, relaxation is not total. Several attempts to reduce the residual stress by introducing stress-compensating layers of $\mathrm{GaP}^{[47,48]}$ or GaNAs ${ }^{[49]}$ between the QDs have been carried out by several groups. Other materials or strategies to improve the performance of the QD IB cells have also been used. $^{[50-52]}$ The data by Hubbard et al., ${ }^{[46]}$ presented in Figure $6 c$, are very interesting. These cells do not have any antireflective coating but they are measured at a higher irradiance conditions (AM0). It is remarkable that they only use 5 layers of QDs in contrast with the 50 layers used by others, underlining the importance of adequate stress reduction.

However, achieving good sub-bandgap quantum efficiency (such as the one in Fig. 4e) is not a good figure of merit for an IB solar cell. Ideally, only the concourse of two sub-bandgap photons should lead to an electron-hole pair. Routine quantum efficiency measurements, when carried out in the absence of bias light, essentially involve observing the photocurrent response of the solar cell to monochromatic light. If some sub-bandgap current is seen under monochromatic illumination in such measurements, the following mechanisms may occur (given the photon energies usually involved): i) the $\mathrm{VB} \rightarrow \mathrm{IB}$ transition is promoted by absorption of one photon, whereas the $\mathrm{IB} \rightarrow \mathrm{CB}$ transition is promoted by thermal escape or field-aided tunneling, and thus preventing, for thermodynamic reasons, the extraction of a voltage above the VB-IB gap (like in QW cells) or ii) two photons of the same energy promote the $\mathrm{VB} \rightarrow \mathrm{IB}$ and $\mathrm{IB} \rightarrow \mathrm{CB}$ transitions. Although these cases would correspond to a genuine IB solar-cell mode of operation with the output voltage still limited by the VB-CB gap, this mode of operation would not be ideal because much of the excess photon energy beyond the 

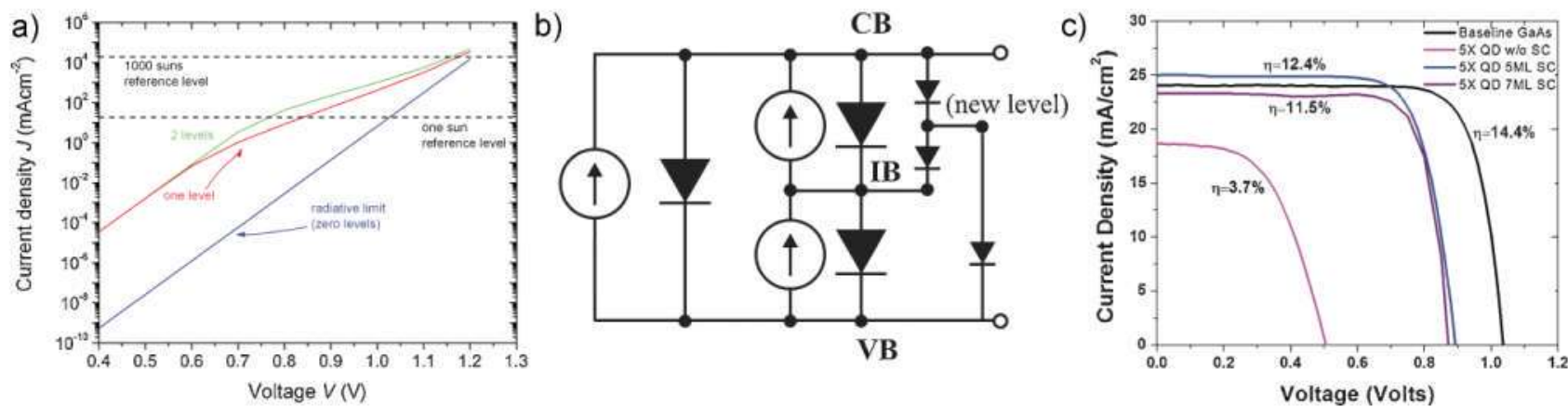

Figure 6. a) Analysis of the dark current-voltage characteristic of an IB solar cell of $1.3 \mathrm{eV}$ total bandgap for several cases, which differ in the number of additional energy levels that are introduced in the energy bandgap. The blue line (zero levels) corresponds to the radiative limit of a single gap solar cell and is shown for reference. The red line (one level) corresponds to the ideal IB solar cell with the IB located at $0.3 \mathrm{eV}$ from the CB. The green line (two levels) corresponds to the case in which one additional energy level exists in-between the IB and the CB (adapted with permission from [10], copyright 2008, Elsevier). This case can be modeled by introducing additional diodes (b) to the IB circuit model to count for the carrier recombination paths introduced by these additional levels (adapted with permission from [10], copyright 2008, Elsevier). The dashed horizontal lines show the current levels corresponding to the photocurrent generated at different illumination levels. Their intersection with the dark current curves determines (assuming the superposition principle fulfilled) the open circuit voltage. As can be seen, the open circuit voltage becomes less dependent on the number of additional energy levels introduced as the concentration increases. c) The one sun AMO (extraterrestrial spectrum and power flux) current density versus voltage curves for the baseline $\mathrm{p}-\mathrm{i}-\mathrm{n}$ GaAs cell and QD-enhanced $\mathrm{p}$-i-n cells both with and without stress compensation (SC) (reproduced with permission from [46], copyright 2008, IEEE).

energy that is necessary to produce the $\mathrm{IB} \rightarrow \mathrm{CB}$ transition would be lost. In addition, mechanism ii) is not likely to occur because the overlap between a localized wavefunction at the QD and an extended wavefunction well above in the $\mathrm{CB}$ is very small, and the corresponding transition matrix element must be very small. However, it still might be observed in sensitive experiments, ${ }^{[43]}$ but it would not be relevant for practical systems. Thus, most probably mechanism i) will occur, which allows the extraction of a sub-bandgap bandgap photocurrent and, therefore, only at high direct bias their quasi-Fermi levels might split. ${ }^{[44]}$ In any case, these observations allow us to conclude that the $\mathrm{VB} \rightarrow \mathrm{IB}$ light absorption must be high.

The QDs' electronic wavefunctions are large enough to permit rather high electric dipole matrix elements despite their low density (at most $10^{17} \mathrm{~cm}^{-3}$ ) compared to that of the atoms in the lattice (in the range of $5 \times 10^{22} \mathrm{~cm}^{-3}$ ). Quantum calculations ${ }^{[40]}$ of the absorption coefficients are in the range of several thousands of $\mathrm{cm}^{-1}$, about 10-50 times less than a bulk material, but still reasonably high. In fact, most of the $\mathrm{VB} \rightarrow \mathrm{IB}$ absorption is excitonic, that is, it takes place between localized states in the QD at the VB (the VB offset further discussed in Section 3.4.3) and the localized states in the IB. The corresponding absorption roughly follows the shape as shown in Figure $2 \mathrm{~b}$ rather than the one in Figure 2c.

The cell presented by Okada ${ }^{[53]}$ with very high density of QDs, shows an excellent current improvement of $23 \%$ with respect to the GaAs cell they used as a comparison (with a good efficiency of about $16 \%$ ). However, interpretation of the cause of the current gain is difficult due to the presence of the GaNAs layers they use to reduce stress, and which have a lower bandgap than the GaAs cell.

Nevertheless, the sensitive two-photon experiment ${ }^{[43]}$ does in some cases not yield any two-photon current. This can only be explained by the absence of $\mathrm{IB} \rightarrow \mathrm{CB}$ absorption, which results either from a small matrix element or because the IB is empty of electrons, instead of being partially filled as required for good IB cell operation. Many scientists are reluctant to dope the QD region to avoid imperfections. If the IB is not partially filled, pumping a second photon will be very difficult and the voltage will be limited by the position of the IB position rather than by the position of the CB. If the thermal escape is effective, this cell will behave then like a QW solar cell, providing bandgap tuning but not an IB effect. Under the QW mode of operation, the confined levels must be empty to facilitate the absorption of photons from the VB.

Finally, some concern about the $\mathrm{IB} \rightarrow \mathrm{CB}$ optical transition being forbidden in QDs may come to mind. Actually, this transition is forbidden in QWs for incident photons normal to the QW planes ${ }^{[54]}$ (the cell surface plane) although the absorption for incident photons laterally is very high. ${ }^{[55]}$ The feasibility of quantum dot infrared photodetectors (QDIPs), ${ }^{[54,56]}$ which in our terms exploit the $\mathrm{IB} \rightarrow \mathrm{CB}$ transition for normal incident photons, demonstrate that this transition is possible. Furthermore, QDIPs are doped in order to fill the IB and allow the $\mathrm{IB} \rightarrow \mathrm{CB}$ transition, supporting our previous argumentation. It is nevertheless possible that the sometimes observed lens-shaped QDs are closer to a QW, and because of this, the devices exhibit small matrix elements for the desired transitions. ${ }^{[57]}$

Diffractive light confinement might be used to improve the $\mathrm{IB} \rightarrow \mathrm{CB}$ transition if it is weak.

\subsubsection{The Issue of the Voltage}

The increase of current achievable with IB solar cells must be accompanied by a conservation of the voltage. In experiments it is common to use a GaAs reference cell and then compare its performance against the results of the cells containing QDs. As indicated in Figure $7 \mathrm{a}$, this is in part an unfair comparison. When the QDs are implemented, the $\mathrm{CB}-\mathrm{VB}$ GaAs bandgap is reduced: first, by the InAs wetting layer (actually a QW) that is formed 
before the QDs start to shrink and which contains a continuous of levels labeled by a $k$-vector parallel to the growth plane; second, by the multiplicity of confined levels appearing in the VB, which are very closely packed due to the high hole-effective mass. Popescu et al. ${ }^{[58]}$ have calculated that the VB confined levels in the InAs/ GaAs cells are as large as $212 \mathrm{meV}$ (Fig. $7 \mathrm{~b}$ ) and can be considered as continuous because each level differs from the neighboring ones less than the optical phonon energy, therefore, they can be considered part of the VB. Near the CB, there also is a continuum of levels that includes (but possibly exceeds) the wetting layer, extending for some $70 \mathrm{meV}$. All these data have been calculated at $0 \mathrm{~K}$ and can become about $7 \%$ smaller at room temperature. They are dependant of the specific QD shape and size. This is to be compared to the reduction of the open-circuit voltage observed in Figure $4 \mathrm{~d}$ of about $180 \mathrm{mV}$ (which refers to the IB cells ${ }^{[46]}$ with, as far as we know, the highest open circuit voltage so far published). It seems very likely that this reduction is almost entirely due to the reduction of the nanostructured material's bandgap, although the always present recombination mechanisms, at least the radiative recombination considered in the detailed balance calculations (Fig. 6a), may ultimately play a role.

The reduction of the bandgap in the cell with QDs should translate into a decrease of the absorption edge corresponding to the $\mathrm{CB} \rightarrow \mathrm{VB}$ transitions in the $\mathrm{QE}$ spectrum for wavelengths below $880 \mathrm{~nm}$, which is the GaAs edge of absorption. However, it is important to note that most of the $\mathrm{CB} \rightarrow \mathrm{VB}$ absorptions occur at the emitter, which is made of conventional GaAs. Sometimes this sub-bandgap absorption is attributed to the wetting-layer absorption and this indeed contributes to the current enhancement observed in practical QD cells, see for example the one shown in Figure 4e.

According to the cited calculations (see Fig. 7b), there are three distinct QD levels in the InAs/GaAs, separated from the mentioned continua. These energy levels are confirmed in our experimental photo-reflectance studies ${ }^{[41,59]}$ at room temperature (see Fig. 7c) at positions close to the calculated ones (the peak marked as QD0 is believed to correspond to a different set of QDs, the seed QDs, also appearing during the growth).

The energy levels introduced in the bandgap by the QDs provide an additional path for recombination, which tends to reduce the voltage, as shown in Figure $6 \mathrm{a}$. Notice that the higher the number of levels existing, the higher the voltage has to be, and therefore the higher the light concentration at which the cells have to be operated in order to overcome the recombination through these levels.

It is worth mentioning that this reduction of bandgap induced by the QDs is not a real fundamental drawback. It can be compensated by using a large bandgap barrier material, e.g., a)

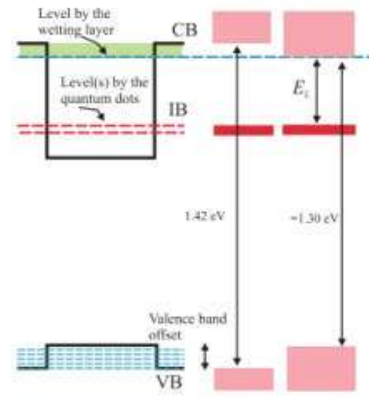

c)

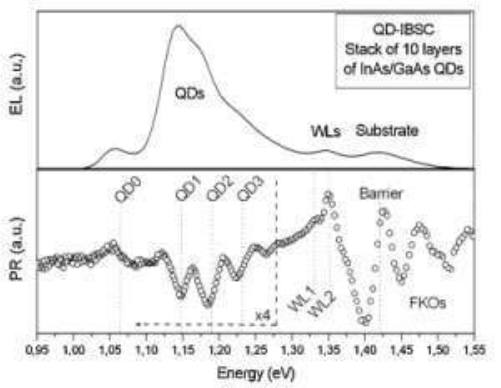

b)

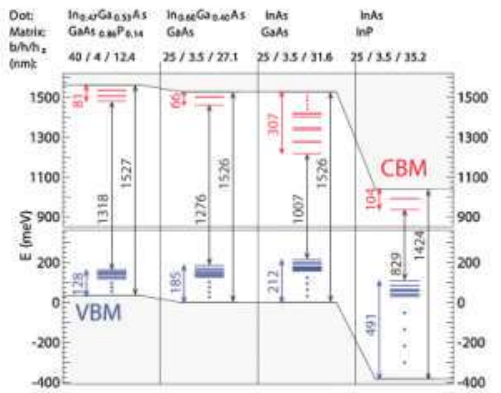

Figure 7. a) Illustration of the reduction of the total bandgap in a QD solar cell as a consequence of the existence of a wetting layer and a VB offset (adapted with permission from [10], copyright 2008, Elsevier). b) Calculated positions of the energy levels in several QD/matrix materials (reproduced with permission from [58], copyright 2008, APS). c) Determination of the confined levels in the InAs/GaAs system by photo-reflectance and electroluminescence measurements (reproduced with permission from [59], copyright 2008, WIP). d) Electronic structure across the $\Gamma \rightarrow X$ path of the first Brillouin zone of vertically spaced QD layers of square-based truncated pyramidal dots for the following cases: left, $h=3 \mathrm{~nm}, b=6 \mathrm{~nm}, L_{x}=20 \mathrm{~nm}$; center, $h=6 \mathrm{~nm}$, $b=12 \mathrm{~nm}, L_{x}=20 \mathrm{~nm}$; right, $h=3 \mathrm{~nm}, b=20 \mathrm{~nm}, L_{x}=40 \mathrm{~nm}$, where $h$ is the height of the truncated pyramidal dot, $b$ is the length of the base, and $L_{x}$ is the distance between dots. Horizontal arrows mark the bottom of the CB in the QD and the bottom of the CB in the barrier region (reproduced with permission from [60], copyright 2006, WIP). e) Energy levels $\left(E_{\mathrm{nI}}\right)$ in a spherical dot under the effective mass approximation. $R$ is the radius of the dot and $V_{0}$ the depth of the potential well. Blue lines correspond to the order $n=1$ and purple lines to $n=2$, respectively. Connected lines correspond to $s$ symmetry (angular momentum $I=0)$, dashed to $p$ symmetry $(I=1)$, dots to $d$ symmetry $(I=2)$, and dashed-dots to $f$ symmetry $(I=3)$, 
some $\mathrm{Al}_{x} \mathrm{Ga}_{1-x} \mathrm{As}$ instead of $\mathrm{GaAs}$ to compensate the bandgap reduction to come when the QDs are formed.

\subsubsection{Best Results So Far}

If high efficiency is the measure of the best cell, the highest efficiency that is published so far to the best of our knowledge is $18.3 \%$ by Blokhin and co-workers, ${ }^{[61]}$ although this efficiency is lower than the one of the GaAs cell the used as a comparison because the observed increase of the current is small and an important voltage reduction takes place. However, the reference cell has an efficiency of $23.77 \%$, which is very high. The highest open-circuit voltage of almost $0.9 \mathrm{~V}$ (at AMO) is the one published by Hubbard et al., ${ }^{[46]}$ and the highest current increase compared to the GaAs test cell (a 23\% increase, which corresponds to a current density $24.26 \mathrm{~mA} \cdot \mathrm{cm}^{-1}$ ) is the one by Okada ${ }^{[53]}$ (University of Tokyo). However, as said before, no clear proof has been provided so far that these cells work under IB principles. Most probably all of them show very strong thermal contact between the IB and CB, which is difficult to avoid for an IB so close to the $C B$.

\subsubsection{Looking for the Ideal QD Material}

As already mentioned, before we started our experimental work, we tried to find optimal dot-barrier materials for high efficiency IB cells. ${ }^{[34,37]}$ Other researchers have based their theoretical work in trying to prevent the appearance of confined states in the VB. ${ }^{[52,62,63]}$ The existence of some offset in the VB, however, can preserve the photon absorption associated with excitonic $\mathrm{VB} \rightarrow \mathrm{IB}$ transitions by maintaining the overlap between the electron wavefunction of these states. Techniques used in the field of QDIPs, such as for example the use of AlGaAs barriers, ${ }^{[64-66]}$ can be used to increase the absorption associated with transitions from the IB to the CB by again increasing the overlap between electron wavefunction at the IB and CB. It is also conceivable that simultaneous overlapping of the electron wave functions in all the bands is achieved naturally by sufficiently increasing the dot density. ${ }^{[67]}$

A serious theoretical-experimental work has been published by Popescu et al. ${ }^{[58]}$ leading to the conclusion that the current repertoire of $\mathrm{GaAs}_{1-x} \mathrm{P}_{x}$ barrier materials and $\mathrm{In}_{y} \mathrm{Ga}_{1-\gamma} \mathrm{As}$ dot materials does not satisfy the ideal energetic locations for the IB. In addition, when the dot size becomes too big, a continuum of energy levels appears between the IB and CB, which practically converts the QD into a quantum well. Dots have to be small to exhibit real dot properties. This is clearly seen in the calculations by Tomic and co-workers using $k \cdot p$ quantum calculations ${ }^{[60]}$ (Fig. 7d).

To further clarify this point, we have carried out calculations for a spherical potential well using the effective mass approximation (Fig. 7e). It is found that for combinations of dot radius $R$ and the potential's depth $V_{0}$ that satisfy $2 R^{2}\left|V_{0}\right| m^{*} / h^{2}<9.87\left(m^{*}\right.$ being the effective mass of the electrons in the band), only one energy level appears. For $V_{0}=1 \mathrm{eV}$ and $m^{*}=0.06 m_{0}$, for example, this leads to a dot radius of $R<3 \mathrm{~nm}$. The corresponding position of the confined energy, $E_{n 1}$, level satisfies $2 R^{2}\left|V_{0}\right| m^{*} / h^{2}=4.516$, which leads to $E_{\text {nl }}$ being located at $0.319 \mathrm{eV}$ below the CB edge. As soon as the radius grows, a multiplicity of levels appears, which in practice can degenerate into a full coverage of the IB-CB energy sub-bandgap. In spite of this, the appearance of a second level that is close to the CB continuum could be beneficial (at the cost of reducing the $\mathrm{CB}-\mathrm{IB}$ bandgap) in order to increase the overlap between the wavefunctions of the electrons in the IB and CB and therefore increase the photon absorption between these two bands.

\subsubsection{Operation in Multijunction (MJ) Configuration}

IB solar cells do not necessarily have to be designed for the full solar spectrum. As matter of fact, when IB cells have to compete with $\mathrm{MJ}$ cells, it might be possible that they preferably are produced in tandem. A tandem of two ideal IB cells is almost as good as a regular ideal six-junction $\mathrm{MJ}$ cell. ${ }^{[68]}$ Taking this into consideration, IB solar cells could then operate in tandem with other regular or IB solar cells whose bandgaps can be optimized.

\section{Bulk Intermediate Band Materials}

\subsection{The Interest of Bulk Intermediate Band Materials}

At the beginning of the research on IB cells, while the concept was in general considered very interesting, the possibility of the existence of actual materials exhibiting an IB was considered improbable. Today it is clear that such materials are possible, starting with the impurities producing deep levels in semiconductors that, when introduced in high concentration (possibly beyond the thermodynamic equilibrium but still stable) may produce IB materials with reduced non-radiative recombination.

These materials are important, because they may support a higher density of IB states and therefore may lead to stronger sub-bandgap photon absorption. It has been seen that the weak absorption has so far been one of the reasons for the low efficiency of the QD IB solar cells. However, this statement has to be considered with some caution as we shall see next.

\subsection{Absorption in Bulk and QD Materials}

We will discuss now the impact of the density of states on the absorption coefficient. This coefficient, $\alpha\left(\mathrm{cm}^{-1}\right)$, is the transition rate $w\left(\mathrm{~s}^{-1}\right)$ divided by the speed of the light in the medium $c / n_{\mathrm{r}}$ ( $n_{\mathrm{r}}$ index of refraction). The transition rate for the dominant dipole electric transitions is proportional to the square of the matrix element of the position vector projection on the photon polarization vector (i.e., to $N|\langle\varphi|\boldsymbol{u} \cdot \boldsymbol{r}| \psi\rangle|^{2}$ where $\varphi$ and $\psi$ are the initial and final electron wavefunctions, $\boldsymbol{r}$ is the position of the electron, $\boldsymbol{u}$ is the light polarization vector and $N$ the number of electrons in a given volume, say the unity volume). The term $|\langle\varphi|\boldsymbol{u} \cdot \boldsymbol{r}| \psi\rangle|$ has the dimension of length and it certainly takes on a value that cannot exceed the span of the wavefunctions involved. We will refer to it as $\delta$. In the calculation of the transition rate it appears as the square, and therefore it can be interpreted as a capture section $\sigma=\delta^{2}$ for the transition. The transition rate is also proportional to the density $N$ of electrons represented by the wavefunction, $\varphi$, able to experience the transition (usually one or per impurity producing the IB or per $\mathrm{QD})$.

Only for the purpose of estimating $\delta$, let us consider $N_{0}$, the highest possible density of impurities or QDs. In the case of the 
impurities, this limit will correspond to the density of atoms in a solid. In the case of the QDs, the limit will correspond to a situation where the QDs touch each other. The volume associated with each impurity or QD in this limiting density is $\left(1 / N_{0}\right)=\gamma \delta^{3}$ where $\gamma$ is a factor close to one but not necessarily below one. The actual density of impurities will be $N=C N_{0}$ with $C$ being smaller than one.

With the preceding nomenclature, the absorption coefficient will be proportional to $N \delta^{2}=N / \gamma^{2 / 3} N_{0}^{2 / 3}=C N_{0}^{1 / 3} / \gamma^{2 / 3}$. Notice that $\gamma^{2 / 3}$ is a factor close to one that depends on the nature of the material. $N_{0}$ also depends on the nature of the IB material. $C$ is one or smaller and reflects the dilution of the IB absorbers. For highest absorption, this factor should be as high as possible.

In the case of solids, the wavefunctions are usually delocalized, e.g., Bloch functions extended across the whole crystal, but even so, $\delta$ is not very large. Far from the origin of coordinates, $r$ varies slowly with respect to the variations of the wavefunctions and since the functions $\varphi$ and $\psi$ are orthogonal, the integral of $\varphi^{*} \psi$ tends to be zero in these regions of the space. Thus $\delta$ remains of the order of the atom spacing, maybe larger than in the case of localized wavefunctions but much smaller than the large extension of the distributed functions. In our nomenclature this is reflected in the value of $\gamma$ that, even if large, will probably not be much larger than 2 or 3 .

Let us now apply this analysis to compare QD and bulk IB materials. For a bulk material, $N_{0}$ is in the range of $5 \times 10^{22} \mathrm{~cm}^{-3}$, which is approximately the density of atoms in a semiconductor. In the case of QDs, it depends on the QD size, but for the optimum size we calculated $d^{[37]}$ that $N_{0}$ is $1.20 \times 10^{18} \mathrm{~cm}^{-3}$ for spherical QDs, assuming that the dots touch each other (in a simple cubic arrangement). Assuming $C=\gamma=1$, the ratio of absorption coefficients is $\left(5 \times 10^{22} / 1.20 \times 10^{18}\right)^{1 / 3}=34.67$.

It is unlikely that the impurity IB atoms, even in high concentration alloys, have a $C$ value much higher than $5 \%$. In some cases, it will be $1 \%$ or even lower. Also the QDs will have a dilution not far from $10 \%$ in the best case. It should be concluded that the absorption coefficient in alloys, provided they have a high density of IB atoms, might be about one order of magnitude above the one in QDs. But it might be smaller if the impurity is very diluted.

\subsection{Synthesizing Bulk IB Materials}

\subsubsection{Highly Mismatched Alloys}

III-V and II-VI alloys normally used for solar cells, where a small amount of V or VI anions are replaced with the isovalent $\mathrm{N}$ and $\mathrm{O}$, respectively, are examples of highly mismatched alloys (HMAs). The unusual properties of HMAs are explained by the band anti-crossing (BAC) model. ${ }^{[69]}$ According to this model, the electronic structure of the HMAs is determined by the interaction between localized states associated with $\mathrm{N}$ or $\mathrm{O}$ atoms and the extended states of the host semiconductor matrix. The BAC model predicts that a narrow band can be formed within the bandgap if the localized state levels occur well below the CB edge. Such a case is realized in $\mathrm{ZnTe}, \mathrm{MnTe}$, and $\mathrm{Zn}_{1-\gamma} \mathrm{Mn}_{\gamma} \mathrm{Te}$ alloys where the $\mathrm{O}$ level is located roughly $0.2 \mathrm{eV}$ below the $\mathrm{CB}$ edge. In particular, alloys of the last family, such as
$\mathrm{Zn}_{0.88} \mathrm{Mn}_{0.12} \mathrm{O}_{0.01} \mathrm{Te}_{0.99}$, have been prepared ${ }^{[70]}$ by implanting $\mathrm{O}$ ions, followed by pulse laser melting and then a rapid thermal annealing process. For a total bandgap of about $2.3 \mathrm{eV}$ (close to the optimum), an IB band is situated at $2.06 \mathrm{eV}$ from the VB as demonstrated by photo-reflectance measurements. Also the $\mathrm{GaN}_{x} \mathrm{As}_{1-x-\gamma} \mathrm{P}_{Y}$ for $y>0.3$ and $x=0.003-0.01$ is a three-band semiconductor alloy with potential applications for highefficiency IB solar cells. ${ }^{[71]}$

A ZnTe:O solar cell with an estimated oxygen concentration of $10^{19} \mathrm{~cm}^{-3}$ has recently been published. ${ }^{[72]}$ Although the efficiency of this cell is still low, it does operate under IB solar cell conditions. This ZnTe cell has a bandgap of about $2.2 \mathrm{eV}$ that is essentially unmodified by the addition of the oxygen, which introduces a level at about $500 \mathrm{meV}$ below the CB. Two-photon operation at room temperature is clearly demonstrated with two light sources of different wavelength. Ordinary quantum efficiency resulting from the photocurrent response of the solar cell to monochromatic light is observed in these cells. In this case the hypothesis of double photon pumping, discussed in Section 3.4.2, with two photons of the same energy is not to be discarded as thermal escape is unlikely. Although the overall efficiency of these devices is small (below 1\%), they demonstrated a $100 \%$ increase in short-circuit current, 15\% decrease in open-circuit voltage, and a $50 \%$ increase in power conversion with respect to the undoped ZnTe cell (Fig. 8a).

\subsubsection{Transition Metals Derived From Ab Initio Calculations}

Since 2002, ab initio band calculations in our group have suggested that certain transition metals could form an IB in III-V ${ }^{[75]}$ compounds. More recently, V-added $\mathrm{In}_{2} \mathrm{~S}_{3}$ with high concentration of $\mathrm{V}\left(\mathrm{V}_{2} \mathrm{In}_{14} \mathrm{~S}_{24}\right)$ has been prepared by solvo-thermal synthesis. ${ }^{[73]}$ Calculations predicted a half-filled band well separated from the valence and CBs with strong sub-bandgap absorption as required by a good IB solar cell. ${ }^{[76]}$ Measurements of the absorption (Fig. 8b) agree rather well with calculations, and both the bandgap and sub-bandgaps are also rather well-situated. Unfortunately, the method of synthesis is impractical for cell manufacturers so that probably a new preparation method will have to be developed to integrate this material in a cell production process. However, $\operatorname{In}_{2} \mathrm{~S}_{3}$ is already being used as a window material in chalcopyrite solar cells.

It is important to stress that ab initio calculations for IB materials in our group are always oriented to materials whose IB is naturally half-filled. In other cases, "half-filling" can be achieved by doping with another material, which in some cases may add more difficulties.

\subsubsection{Thin Film Technology for Intermediate Band Cells}

Intermediate band materials have been sought with the specific idea of being integrated in present chalcopyrite solar-cell processing. It was found that the best candidate for ideal IB cells among those commonly used in this technology for one-sun operation is $\mathrm{CuGaS}_{2}$. The efficiency versus the position of the IB level is then calculated again for the ideal cell for that material bandgap. The position of the level for a given impurity is calculated based on the fact that for III-V and II-VI semiconductors its position measured for the vacuum level is the same, no matter which is the host. ${ }^{[77,78]}$ This concept is also assumed to apply for (I,III) $\mathrm{VI}_{2}$ chalcopyrites. Many of these 
a)
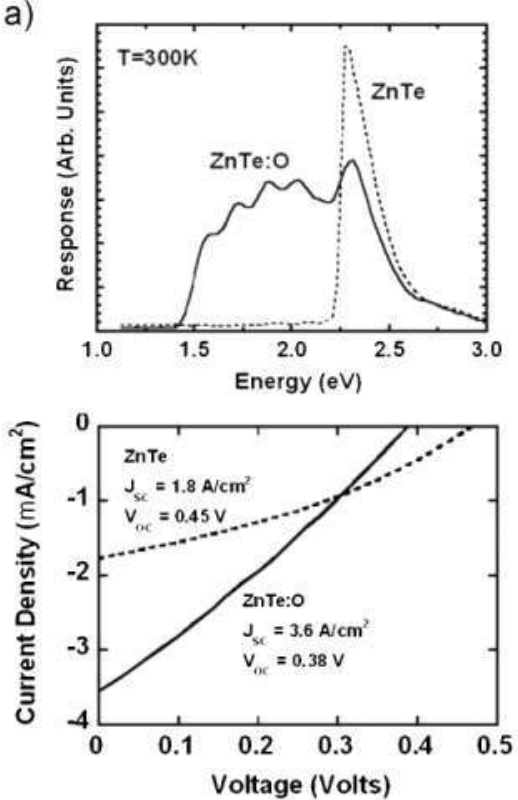

b)

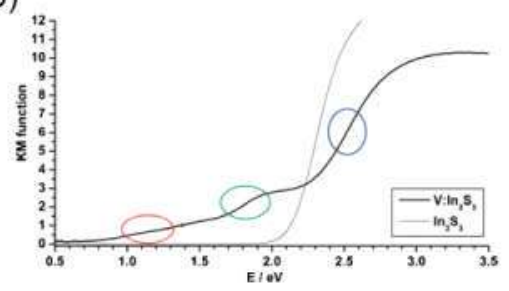

c)

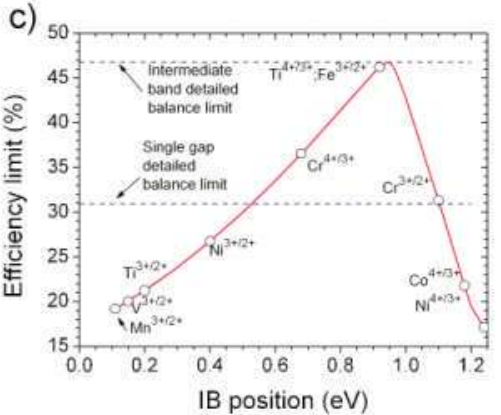

Figure 8. a) Photoresponse (top) and current-voltage characteristic (bottom) of a $\mathrm{ZnTe}: \mathrm{O}$ based IBSC compared with a ZnTe reference (reproduced with permission from [72], copyright 2009, AIP). b) K-M function (absorption) measurements versus photon energy for $V_{2} I_{14} S_{24}$ and $I_{2} S_{3}$ obtained by solvothermal synthesis (reproduced with permission from [73], copyright 2008, ACS). The red circle is associated with transitions from the IB to the CB, the green one to transitions from the VB to the IB, and the blue one to transitions from the VB to the CB. c) Detailed balance efficiency prediction at one sun for IB solar cells based on $\mathrm{CuGaS}_{2}$ with some transition elements inserted (adapted with permission from [74], copyright 2008, AIP).

materials are expected to be very compatible with thin-film cell technology. Fe and Ti seem to be the most promising impurities (Fig. 8c).

\subsubsection{Intermediate Bands Generated From Deep Levels}

As already mentioned, Si samples that are heavily doped with $\mathrm{Ti}$ have been prepared ${ }^{[28]}$ by ion implantation (with densities above $2 \times 10^{21} \mathrm{~cm}^{-3}$ ) followed by a pulsed-laser melting process that restores high quality to the crystal. At low temperatures, a rectifying junction is formed between the Ti-doped layer and the highly resistive $\mathrm{n}$-substrate, which can be interpreted as an IB-nSi junction. ${ }^{[79]}$ Under these low-temperature conditions at which the Ti-doped layer becomes electrically isolated from the substrate, the Ti-doped layer seems to show p-mobility, which is attributed to the almost electron-filled Ti level that would act as an IB. As we already described, the high Ti concentration seems to present a reduced non-radiative recombination ${ }^{[24]}$ by the electron wavefunction delocalization due to high concentration.

\section{Conclusions}

We have presented here the current state of research on the IB solar cell. Despite the high theoretical efficiency potential $(63 \%)$ of the IB solar cells, so far no cell has produced efficiencies that would make them practical for commercial use. However, the IB basic principles have been experimentally proven..$^{[43,44,72]}$ Experimental evidence has also been produced to confirm the reduction of nonradiative recombination in heavily doped IB materials once the electrons are delocalized in it, and a real band - rather than impurity levels has been obtained. ${ }^{[24]}$

Most experimental efforts have been realized in the field of InAs/GaAs QD solar cells, whose bandgaps are very far from the optimal ones. Nevertheless, the efficiencies achieved are below expectations. The reasons for the poor efficiencies are insufficient current increase and excessive voltage reduction. The poor current increase is partly overcome by the growth of more QD layers, once stress-balanced growth techniques are developed.

Concerning the reduction of the voltage, the main cause is the reduction of the effective bandgap due to the existence of the QDs and the associated VB offset and wetting layers. This is not a real limitation and comparison of the InAs/ GaAs solar cell with the same GaAs cell without QDs is unfair. Actually, the comparison with a GaAs solar cell would only be valid if an $\mathrm{Al}_{x} \mathrm{Ga}_{1-x} \mathrm{As}$ alloy that compensates the reduction of the effective bandgap would be used as the barrier material when the QDs are manufactured.

A more serious problem is that, so far, a very strong $\mathrm{IB} \rightarrow \mathrm{CB}$ thermal escape is produced so that the voltage cannot exceed the $\mathrm{VB} \rightarrow \mathrm{IB}$ bandgap. In addition, the IB $\rightarrow \mathrm{CB}$ light absorption seems to be very small and this would make a voltage above the $E_{\mathrm{FI}}-E_{\mathrm{FV}}$ splitting level impossible. However, it is possible that solutions are found to prevent both problems. If this were to be the case, the InAs/GaAs cell may be rather efficient in concentration. However, the search for a new QD-barrier couple of materials, whose bandgaps are closer to the optimal configuration, and thus have the IB more separated from the $\mathrm{CB}$, might perhaps be the fastest way towards a practical solution. Many scientists are trying to optimize the cell quantum efficiency under monochromatic illumination. We have stressed that this technique does not allow the identification of the two-photon mechanism with which sub-bandgap currents and CB-VB limited voltage can be achieved. One of the problems often observed is the reluctance of researchers to add donor atoms to half-fill the IB, and thus making second photon $\mathrm{IB} \rightarrow \mathrm{CB}$ transitions very difficult.

A number of bulk-based IB materials have already been developed. Highly mismatched II-VI and III-V alloys have proven to produce such IB materials, ${ }^{[0,71]}$ and a solar cell ${ }^{[72]}$ has been presented that clearly demonstrates IB operation with an $100 \%$ increase in short-circuit current, $15 \%$ decrease in opencircuit voltage, and $50 \%$ overall increase in power conversion, although the efficiency is still below $1 \%$.

IB materials have also been found in some transition metals in indium thiospinels where the IB is naturally half-filled. ${ }^{[76]}$ Lifetime $^{[24]}$ and Hall effect ${ }^{[79]}$ experiments in Ti-implanted Si 
suggest the existence of an IB; this may open an interesting path for research on deep levels as precursors for IB materials.

In summary, the IB solar cell is an attractive concept, which involves interesting physics. Its development is not trivial but there are several promising paths and much has been learned in about one decade of research. Once this cell is developed, it might be possible to find it in concentrators in tandem configurations with ordinary or other IB cells operating at efficiencies above those of the present MJ cells for concentrator operation or above the present thin-film solar cells for one-sun operation.

\section{Acknowledgements}

This work has been supported by the European Commission within the project IBPOWER (Contract 211640) and the projects NUMANCIA (S-0505/ENE/000370) funded by the Comunidad de Madrid and GENESIS-FV (CSD2006-00004) funded by the Spanish National Program.

Received: July 17, 2009 Published online:

[1] E. A. Alsema, Prog. Photovoltaics: Res. Appl. 2000, 8, 17

[2] T. B. Johansson, H. Kelly, A. K. N. Reddy, R. H. Williams, L. Burnham, Renewable Energy Saurces for Fuel and Electricity, Island Press, Washington, DC 1993.

[3] A. Luque, A. Martí, Phys. Rev. Lett. 1997, 78, 5014.

[4] W. Shockley, H. J. Queisser, J. Appl. Phys. 1961, 32, 510.

[5] G. L. Araujo, A. Marti, Solar Energy Mater. Solar Cells 1994, 33, 213.

[6] A. Luque, A. Marti, Electron. Lett. 2008, 44, 943.

[7] M. Yamaguchi, A. Luque, IEEE Trans. Electron Dev. 1999, 46, 2139.

[8] A. Luque, A. Martí, Prog. Photovoltaics: Res. Appl. 2001, 9, 73.

[9] R. Winston, W. T. Welford, Optics of Nonimaging Concentrators, Academic Press, New York 1979.

[10] A. Marti, E. Antolin, E. Canovas, N. Lopez, P. G. Linares, A. Luque, C. R. Stanley, C. D. Farmer, Thin Solid Films 2008, 516, 6716.

[1]] A. Luque, A. Martí, L. Cuadra, IEEE Trans. Electron Dev. 2003, 50, 447.

[12] A. Luque, A. Martí, C. Stanley, N. López, L. Cuadra, D. Zhou, A. Mc-Kee, J. Appl. Phys. 2004, 96, 903.

[13] L. Cuadra, A. Marti, A. Luque, IEEE Trans. Electron Dev. 2004, 57, 1002.

[14] E. Yablonovitch, G. D. Cody, IEEE Trans. Electron Dev. 1982, 29, 300.

[15] R. Morf, H. Kiess, "Submicron gratings for light trapping in silicon solar cells: a theoretical study", presented at Proc. 9th Photovoltaic Solar Energy Conf, Freiburg 1989.

[16] C. Heine, R. H. Morf, Appl. Opt. 1995, 34, 2476.

[17] 1. Tobias, A. Luque, A. Marti, J. Appl. Phys. 2008, 104, 034502.

[18] S. P. Bremner, C. B. Honsberg, R. Corkish, in 28th Photovoltaics Specialist Conference, IEEE 2000, p. 1200.

[19] A. Luque, A. Martí, Phys. Rev. B 1997, 55, 6994.

[20] A. Luque, A. Martí, L. Cuadra, IEEE Trans. Electron Dev. 2001, 48, 2118.

[21] A. Luque, A. Martí, L. Cuadra, Physica E 2002, 14, 107.

[22] A. Luque, A. Martí, E. Antolín, C. Tablero, Physica B 2006, 382, 320.

[23] D. V. Lang, C. H. Henry, Phys. Rev. Lett. 1975, 35, 1525.

[24] E. Antolin, A. Marti, I. Olea, D. Pastor, G. Gonzalez-Diaz, I. Martil, A. Luque, Appl. Phys. Lett. 2009, 94, 042115.

[25] K. Huang, A. Rhys, Proc. R. Soc. Lond. Ser. A: Math. Phys. Sci. 1950, 204, 406.

[26] N. F. Mott, Rev. Mod. Phys. 1968, 40, 677.

[27] A. M. Green, Prog. Photovoltaics: Res. Appl. 2001, 9, 137.

[28] J. Olea, M. Toledano-Luque, D. Pastor, G. Conzalez-Diaz, I. Martil, J. Appl. Phys. 2008, 104, 016105.
[29] J. W. Chen, A. G. Milnes, A. Rohatgi, Solid State Electron. 1979, 22, 801.

[30] A. Rohatgi, J. R. Davis, R. H. Hopkins, P. Raichoudhury, P. G. McMullin, J. R. McCormick, Solid State Electron. 1980, 23, 415.

[31] A. C. Wang, C. T. Sah, J. Appl. Phys. 1984, 56, 1021.

[32] R. Lago-Aurrekoetxea, I. Tobias, C. del Canizo, A. Luque, J. Electrochem. Soc. 2001, 748, G200.

[33] A. Martí, L. Cuadra, A. Luque, in Proc. 28th IEEE Photovoltaics Specialists Conference, IEEE, New York 2000, p. 940.

[34] A. Martí, L. Cuadra, A. Luque, IEEE Trans. Electron Dev. 2001, 48, 2394.

[35] A. Marti, N. Lopez, E. Antolin, E. Canovas, A. Luque, C. R. Stanley, C. D. Farmer, P. Diaz, Appl. Phys. Lett. 2007, 90, 233510.

[36] A. Luque, A. Martí, N. López, E. Antolín, E. Cánovas, C. R. Stanley, C. Farmer, P. Díaz, J. Appl. Phys. 2006, 99, 094503.

[37] L. Cuadra, A. Martí, A. Luque, C. R. Stanley, A. McKee, in Proc. of the 17th European Photovoltaic Solar Energy Conference, WIP-Renewable Energies and ETA, Munich, Florence 2001, p. 98.

[38] K. W. J. Barnham, B. Braun, J. Nelson, M. Paxman, C. Button, J. S. Roberts, C. T. Foxon, Appl. Phys. Lett. 1991, 59, 135.

[39] T. Kirchartz, K. Seino, J. M. Wagner, U. Rau, F. Bechstedt, J. Appl. Phys. 2009, $105,12$.

[40] S. Tomic, T. S. Jones, N. M. Harrison, Appl. Phys. Lett. 2008, 93, 263105.

[41] E. Cánovas, A. Martí, N. López, E. Antolín, P. G. Linares, C. D. Farmer, C. R. Stanley, A. Luque, Thin Solid Films 2008, 516, 6943.

[42] A. Martí, L. Cuadra, A. Luque, IEEE Trans. Electron Dev. 2002, 49, 1632.

[43] A. Marti, E. Antolin, C. R. Stanley, C. D. Farmer, N. Lopez, P. Diaz, E. Canovas, P. G. Linares, A. Luque, Phys. Rev. Lett. 2006, 97, 247701.

[44] A. Luque, A. Marti, N. Lopez, E. Antolin, E. Canovas, C. Stanley, C. Farmer, L. ). Caballero, L. Cuadra, J. L. Balenzategui, Appl. Phys. Lett. 2005, 87, 083505.

[45] E. Antolín, A. Martí, P. G. Linares, E. Cánovas, D. F. Marrón, A. Luque, Demonstration and analysis of the photocurrent produced by absorption of two sub-bandgap photons in a QD IB solar cell, presented at 23 European Photovoltaic Solar Energy Conference, Valencia 2008.

[46] S. M. Hubbard, C. G. Bailey, C. D. Cress, S. Polly, J. Clark, D. V. Forbes, R. P. Raffaelle, S. G. Bailey, D. M. Wilt, in 33 IEEE Photovoltaic Specialists Conference, IEEE, San Diego 2008.

[47] S. M. Hubbard, C. D. Cress, C. G. Bailey, R. P. Raffaelle, S. G. Bailey, D. M. Wilt, Appl. Phys. Lett. 2008, 92, 123512.

[48] D. Alonso-Alvarez, A. G. Taboada, 1. M. Ripalda, B. Alen, Y. Conzalez, L. Conzalez, J. M. Garcia, F. Briones, A. Marti, A. Luque, A. M. Sanchez, S. I. Molina, Appl. Phys. Lett. 2008, 93, 123114.

[49] R. Oshima, A. Takata, Y. Okada, Appl. Phys. Lett. 2008, 93, 08311 ].

[50] G. Wei, S. R. Forrest, Nano Lett. 2007, 7, 218.

[51] R. B. Laghumavarapu, M. El-Emawy, N. Nuntawong, A. Moscho, L. F. Lester, D. L. Huffaker, Appl. Phys. Lett. 2007, 91, 3.

[52] A. G. Norman, M. C. Hanna, P. Dippo, D. H. Levi, R. C. Reedy, J. S. Ward, in 31st IEEE Photovoltaic Specialists Conference, IEEE, New York 2005, p. 43.

[53] Y. Okada, in MRS Fall Meeting, Symposium N, Boston 2008.

[54] J. Phillips, K. Kamath, P. Bhattacharya, Appl. Phys. Lett. 1998, 72, 2020.

[55] L. C. West, S. J. Eglash, Appl. Phys. Lett. 1985, 46, 1156.

[56] K. W. Berryman, S. A. Lyon, M. Segev, Appl. Phys. Lett. 1997, 70, 1861.

[57] H. C. Liu, J. Y. Duboz, R. Dudek, Z. R. Wasilewski, S. Fafard, P. Finnie, "Quantum dot infrared photodetectors", presented at the int. Conf. on Superlattices Nanostructures and Nanodevices (ICSNN-02), Toulouse, France, July 2002, 631.

[58] V. Popescu, G. Bester, M. C. Hanna, A. G. Norman, A. Zunger, Phys. Rev. B 2008, 78, 205321.

[59] E. Canovas, A. Martí, D. Fuertes-Marrón, E. Antolin, G.-L. P. A. Luque, in Proc. 23th Ruropean Photovoltaic Conference, WIP, Valencia, September 2008, p. 298.

[60] S. Tomic, T. S. Jones, N. M. Harrison, Appl. Phys. Lett. 2008, 93, 674.

[61] S. A. Blokhin, A. V. Sakharov, A. M. Nadtochy, A. S. Pauysov, M. V. Maximov, N. N. Ledentsov, A. R. Kovsh, S. S. Mikhrin, V. M. Lantratov, S. A. Mintairov, N. A. Kaluzhniy, M. Z. Shvarts, Fizika i Tekhnika Poluprovodnikov (Semiconductors) 2009, 43, 537 (in Russian). 
[62] M. Y. Levy, C. Honsberg, A. Martí, A. Luque, in 37th IEEE Photovoltaics Specialists Conf., IEEE, New York, January 2005, p. 90.

[63] M. Y. Levy, C. Honsberg, IEEE Trans. Electron Dev. 2008, 55, 706.

[64] S. W. Lee, K. Hirakawa, Y. Shimada, Appl. Phys. Lett. 1999, 75, 1428.

[65] G. Wei, S. R. Forrest, Nano Lett. 2007, 7, 218.

[66] A. Martí, E. Antolín, E. Cánovas, N. López, A. Luque, C. Stanley, C. Farmer, P. Díaz, C. Christofides, M. Burhan, in Proc. of the 27st European Photovoltaic Solar Energy Conf. (Eds: J. Poortmans, H. Ossenbrink, E. Dunlop, P. Helm), W/P-Renewable Energies, Munich, September 2006, p. 99.

[67] A. Marti, C. R. Stanley, A. Luque, in Nanostructured Materials for Solar Energy Conversion (Ed: T. Soga), Elsevier, Amsterdam, 2006.

[68] E. Antolín, A. Martí, A. Luque, in Proc. of the 27 st European Photovoltaic Solar Energy Conference (Eds: ). Poortmans, H. Ossenbrink, E. Dunlop, P. Helm), W/P-Renewable Energies, Munich, September 2006, p. 412.

[69] W. Walukiewicz, W. Shan, K. M. Yu, J. W. Ager, E. E. Haller, I. Miotkowski, M. J. Seong, H. Alawadhi, A. K. Ramdas, Phys. Rev. Lett. 2000, 85, 1552.
[70] K. M. Yu, W. Walukiewicz, J. Wu, W. Shan, J. W. Beeman, M. A. Scarpulla, O. D. Dubon, P. Becla, Phys. Rev. Lett. 2003, 91, 246403.

[71] K. M. Yu, W. Walukiewicz, J.W. Ager, III, D. Bour, R. Farshchi, O. D. Dubon, S. X. Li, I. D. Sharp, E. E. Haller, Appl. Phys. Lett. 2006, 88, 092110.

[72] W. Wang, A. S. Lin, J. D. Phillips, Appl. Phys. Lett. 2009, 96, 011103.

[73] R. Lucena, I. Aguilera, P. Palacios, P. Wahnon, J. C. Conesa, Chem. Mater. 2008, 20, 5125.

[74] A. Martí, D. F. Marrón, A. Luque, J. Appl. Phys. 2008, 103, 073706.

[75] P. Wahnón, C. Tablero, Phys. Rev. B 2002, 65, 155115.

[76] P. Palacios, I. Aguilera, K. Sanchez, J. C. Conesa, P. Wahnon, Phys. Rev. Lett 2008, 101, 046403.

[77] M. Hamera, W. Walukiewicz, D. D. Nolte, E. E. Haller, Phys. Rev. B 1989, 39, 10114.

[78] M. J. Caldas, A. Fazzio, A. Zunger, Appl. Phys. Lett. 1984, 45, 671.

[79] G. Gonzalez-Daz, J. Olea, I. Martil, D. Pastor, A. Mart, E. Antoln, A. Luque, Solar Energy Mater. Solar Cells 2009, 93, 1668. 